\title{
Clinical Approach for Treating Dilated Cardiomyopathy in a Hog-nosed Skunk (Conepatus chinga)
}

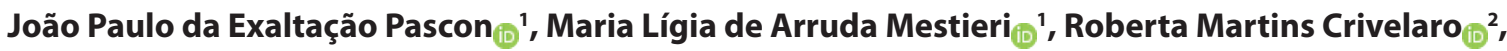 \\ Roberto Thiesen $_{\mathbb{C}^{1}}{ }^{1} \&$ Claudia Acosta Duarte ${ }_{\mathbb{D}^{1}}{ }^{1}$
}

\begin{abstract}
Background: Dilated cardiomyopathy (DCM) is a multifactorial disease characterized by systolic dysfunction of myocardium, affecting domestic animals like dogs, cats, and ferrets. It was sporadically described in non-domestic species, generally as a necropsy observation. The hog-nosed skunk (Conepatus chinga) is a small mammal, carnivore, belonging to the Mephitidae family, found in southern South America, and considered as concerned in a conservation status of the species. The goal of this issue is report the first clinical approach of dilated cardiomyopathy in a young hog-nosed skunk, elucidating the challenging aspects of the diagnostic, therapy, and clinical outcome.

Case: A newborn hog-nosed skunk (Conepatus chinga) was found in a farm of southern Brazil in poor nutritional and behavioral conditions. Thirty days of nutritional supplementation based on cow's milk, fruits and insects were necessary to recover its body weight score and activity level. However, 2 months after adoption, the skunk showed acute dyspnea and abnormal breath sound, decreased appetite, and loss of weight. Firstly, these signs were associated with a possible pulmonary infection or other respiratory disease. However, the general cardiomegaly and pulmonary edema pattern observed on the thoracic radiography, changed the clinical approach, conducting the presumptive diagnosis to congestive heart failure (pulmonary edema) caused by an unknown cardiac disease as a dilated cardiomyopathy (CDM). An adequate physical, echocardiographic, electrocardiographic, and hematological evaluation was possible only after chemical restraint by sevofluorane. Systolic heart murmur and pulmonary cracking sounds were detected on thoracic auscultation. These findings associated to the echocardiography features of four chamber dilatation, systolic (fractional shortening-FS $=9 \%$ and ejection fraction- $\mathrm{EF}=22 \%$ ) and diastolic (mitral E/A ratio $=4.93$ ) dysfunctions, and bilateral ventriculoatrial regurgitation (mitral and tricuspid) were highly correlated with DCM. Considering the etiologies of $\mathrm{CDM}$ described in domestic species and the poor nutritional condition previously observed in the case, nutritional and idiopathic etiologies of DCM were considered for this case. Therapy was based on furosemide at hospital (4 mg/kg subcutaneous, single doses) and home ( $2 \mathrm{mg} / \mathrm{kg}$ orally, BID), enalapril maleate $(0.5 \mathrm{mg} / \mathrm{kg}$ orally, every $48 \mathrm{~h})$, taurine supplementation (100 $\mathrm{mg}$ orally, SID), and pimobendan ( $0.5 \mathrm{mg} / \mathrm{kg}$ orally, BID). Clinical improvement was already observed on the second day of treatment, and monitored for 5 months, when the skunk was completely revaluated. The second echocardiographic exam showed improvement in systolic $(\mathrm{FS}=20 \%$ and $\mathrm{EF}=43 \%)$ and diastolic functions (mitral $\mathrm{E} / \mathrm{A}$ ratio $=2.05)$, tricuspid regurgitation was not observed, and decrease the left atrial and ventricular dimensions were seen. Due to good clinical outcome, furosemide was gradually reduced until complete withdrawal without any clinical complications or worsening. After 30 months of therapy of pimobendan, taurine, and enalapril maleate, the skunk has good quality captive life without congestive heart failure recurrence.

Discussion: The lack of information about DCM in hog-nosed skunk turns the clinical diagnosis and therapy challenging. However, the radiographic and echocardiographic features seamed to lead the same domestic animal patterns. The therapy based on decrease the cardiac overload, increase the myocardial inotropic function (pimobendan and taurine) resulted on excellent clinical and echocardiographic outcome. Although the etiologies of DCM in this species are not stablished, the diagnostic and therapeutic approaches applied resulted on excellent clinical results, and therefore may provide useful information about this cardiac condition in skunk species.
\end{abstract}

Keywords: cardiovascular, disease, Mephitidae, therapy, wild animals.

DOI: $10.22456 / 1679-9216.116202$ 


\section{INTRODUTION}

Dilated cardiomyopathy (DCM) is a primary myocardial disease characterized by reduced contractility and ventricular dilatation involving the left or both ventricles. It is usually observed in dogs, cats [20], and ferrets [28]. In skunks, in a 10-year investigation in a captive environment, only 1 case in Mephitis mephitis [3]. In fact, the lack of standard cardiac measurements in this species and the absence of complete reports of DCM in skunks make diagnosis and therapy challenging. References for diagnosis and therapy are extrapolated from other species, such as domestic ferrets [3]. Hog-nosed skunks (Conepatus chinga) belong to the Mephitidae family [16], and information about cardiac diseases is this specie was not found, so reports showing reference data for diagnosis and/or treatment should be encouraged. The present report aims to describe clinical signs, radiographic/electrocardiographic/echocardiographic features, and clinical outcome after therapy of a young hog-nosed skunk diagnosed with heart failure secondary to DCM.

\section{CASE}

A newborn skunk was found alone and in a poor nutritional condition, by a farm veterinarian. The skunk diet was then based on cow's milk for one month, then fruits were gradually added daily, and insects (crickets) added 3 times a week, resulting in good body score recovery and normal activity level.

However, after 2 months, the skunk suddenly developed dyspnea and abnormal breath sound, decreased appetite, and loss of weight. The animal was referred to a Veterinary Hospital, with a suspect of respiratory disease. The thoracic and abdominal radiographs $^{1,2}$ [Portable Xray: Aquila plus 300, Phillips ${ }^{\circledR}$; Digital reading system: Carestream Directview $\mathrm{CR}^{\circledR}$ ] showed pulmonary edema pattern and global cardiac enlargement (Figure1). However, general anesthesia was necessary to continue the diagnostic exploration by echocardiography, electrocardiography, and blood tests.

Sevoflurane $^{3}$ (Sevocris $^{\circledR}$ ) was used for chemical restraint, delivered in $100 \%$ oxygen through an induction chamber. Once the patient loss righting reflex and was unreactive to stimuli, it was removed from the chamber and anesthesia was continued by face mask.

The anesthesia was indispensable to allow an appropriated physical examination, and for proper information obtained by echocardiography ${ }^{4}$ [MyLab Twice Vet ultrasonographic, Esaote $\left.{ }^{\circledR}\right]$, electrocardi- ography ${ }^{5}$ [vet ECGPC, TEB $^{\circledR}$ ], and hematology tests. Without chemical restraint the thorax auscultation was not possible, however, under anesthesia the left thorax auscultation revealed a low-grade cardiac murmur (II/ VI) and tachycardia (200 bpm).

The echocardiographic evaluation confirmed a 4-chamber heart dilatation, spontaneous blood contrast in the left ventricle (Figure 2A), and impairment of ventricular contraction (Figure 2B). Adding to the clinical signs and nonspecific findings of bidimensional images, the diagnosis of DCM was pointed out. Diastolic dysfunction was also observed by a restrictive pattern of left ventricle filling (Figure 3). The complete structural and functional left ventricle echocardiographic measures of the skunk heart are listed in Table 1.

The electrocardiogram confirmed the normal sinus rhythm without arrhythmias (Figure 4). No alterations in hematological parameters or biochemical dosages (alanina aminotransferase - ALT; urea and creatinine) were seen. Therapy was initiated and included furosemide ${ }^{6}$ at hospital [Lasix ${ }^{\circledR}-4 \mathrm{mg} / \mathrm{kg}$ subcutaneous, single doses) and home [Lasix ${ }^{\circledR}-2 \mathrm{mg}$ / $\mathrm{kg}$ orally, BID], enalapril maleate ${ }^{7}$ Atmos $^{\circledR}-0.5 \mathrm{mg} / \mathrm{kg}$ orally, every $48 \mathrm{~h}$ ], taurine [handling pharmacy - 100 mg orally, SID], and pimobendan ${ }^{8}$ [Vetmedin $^{\circledR}-0.5$ $\mathrm{mg} / \mathrm{kg}$ orally, BID]. The patient demonstrated clinical improvement in the second day of therapy, restoring the respiratory status and the level of activity.

Five months after the diagnosis and onset of the therapy, the skunk showed normal growth and level of activity, without any clinical signs of heart failure. The patient underwent to the same protocol of anesthesia and examinations, to get a comparative data after the established therapy. Low-grade systolic murmur was still noticed. The echocardiographic measurements showed a decrease in heart dimensions and improvement on systolic and diastolic function (Table 1). The electrocardiogram showed respiratory sinus arrhythmia (Figure 5). Once again, no hematological parameters alteration was seen.

Considering the good clinical outcome and echocardiographic response to the therapy, a decrease of furosemide was initiated, with the frequency of administration to once a day. Pimobendan, taurine, and elanapril prescription were maintained. After 15 days, furosemide was prescribed only every $48 \mathrm{~h}$ followed by a complete stop after twenty days. No respiratory distress or activity level decrease was seen until the actual time (30 months after diagnostic). 


\section{DISCUSSION}

Chemical restriction is often necessary for exotic/wild animal proper examination and diagnostic imaging positioning. Sevoflurane was chosen because it has lower arrhythmogenicity when compared with isoflurane and halothane [13], providing rapid induction, recovery, and control of depth of anesthesia [14], and shows potential cardioprotective effects [4]. The physical examination showed tachycardia, using reference range of 140-190 bpm [11]. These clinical signs are commonly observed in skunks with cardiac conditions, mainly when associated with murmur [3].

Skunks were previously referred as affected by myocardial fibrosis and myxomatous valve, and less commonly by degeneration hypertrophic cardiomyopathy and dilated cardiomyopathy [3]. As no specific protocol for skunks were found, the echocardiographic

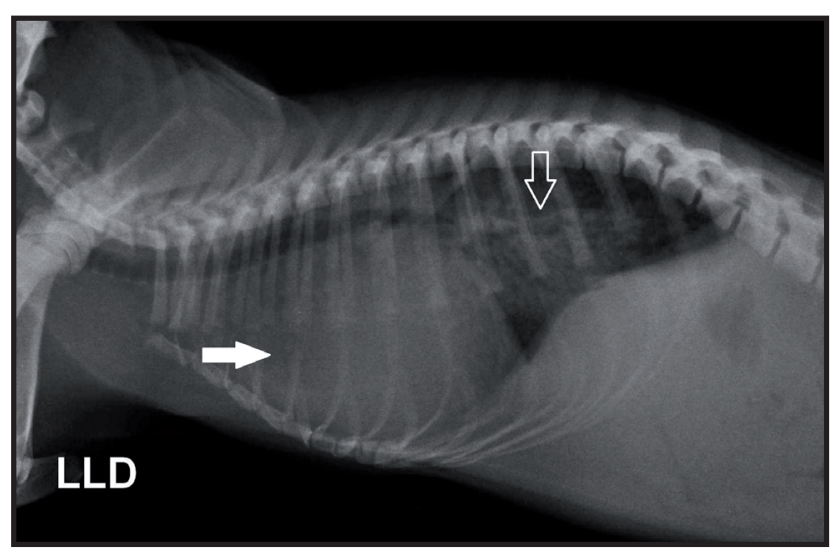

Figure 1. Right lateral (LLD) thoracic radiography showing the cardiomegaly with loss of cranial limits of the heart (full arrow) and pulmonary edema due to left congestive heart failure (empty arrow). examination followed the scanning protocol used in dog and cat [27]. The findings were comparable to those observed in dogs [20], cats [26], and ferrets [6] affected by DCM.

In the author's opinions the combination of respiratory distress, cardiac mumur, cardiomegaly on the radiographic images, and the echocardiographic information of systolic dysfunction, associated to a heart chamber dilatations, and ventriculoatrial regurgitation strongly suggest the diagnostic of DCM in this case. The restrictive pattern of left ventricle filling was also observed in an earlier description of DCM in a skunk species [3], suggesting the occurrence of diastolic dysfunction. However, the lack of standard references for other diastolic measures in skunks, such as left ventricle isovolumic relaxation time (IVRT), flow propagation velocity (Vp), and mitral E/E'ratio made the evaluation and classification of abnormalities challenging. Despite this, the authors believe that those measurements were abnormal.

Once the diagnostic has been defined, the search about possible etiologies of DCM involved, and possible comorbidities became a priority to the authors to prescribe a correct therapy. In this context, the normality of hematological counting according to the reference range for striped skunk [18] helped to ruled out the differential diagnostic of bacterial pneumonia.

The idiopathic is considering the most common etiology of CDM in dogs and ferrets [20,28]. However, genetic factors, toxic factors, taurine deficiency [20], and Trypanosoma cruzi infections [21] are other possible

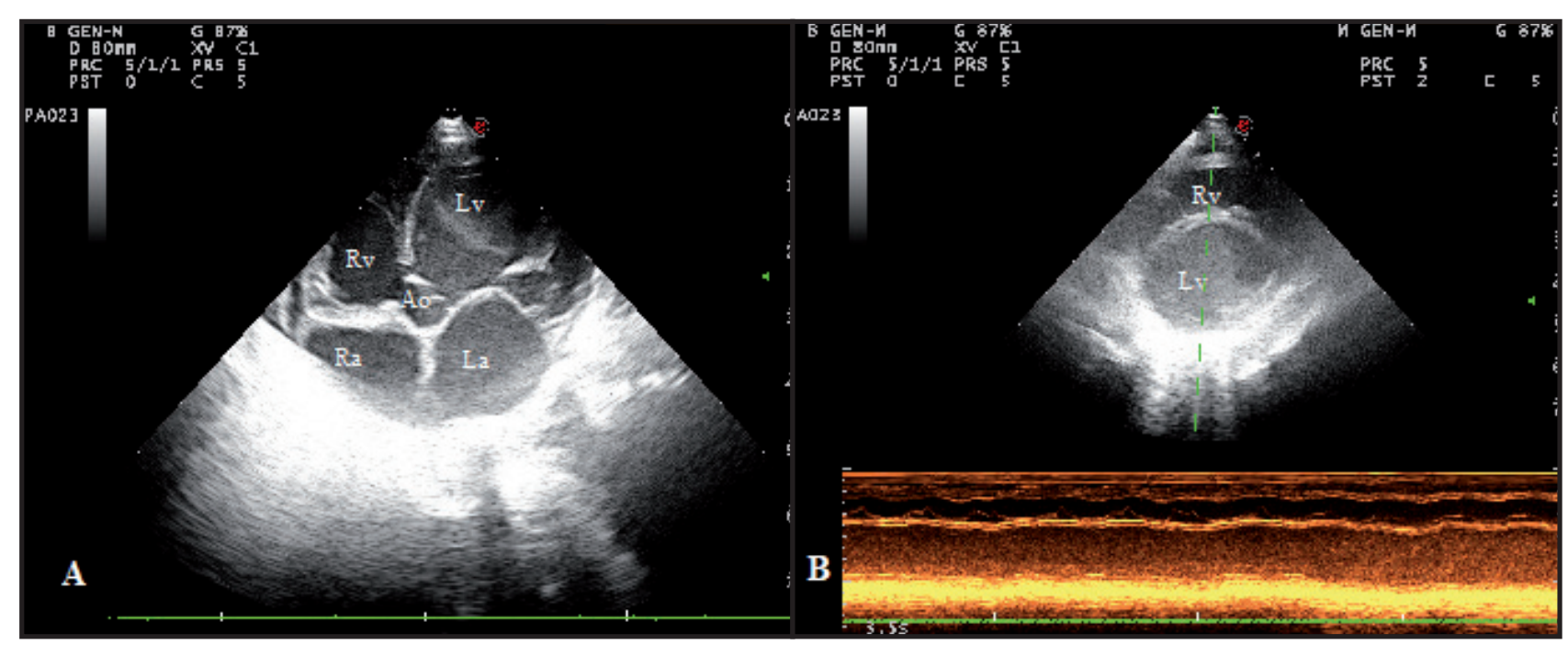

Figure 2. A- Bidimensional echocardiogram, left paresternal window, showing atriums and ventricles chamber enlargements and spontaneous blood contrast seen in the left ventricle, on apical 5-chamber view. B- M-mode echocardiogram, the short axis of the right parasternal view, on the chordal plane, showing a minimal contraction of the left ventricular free wall and interventricular septum. AO: aorta. LA: left atrium. LV: left ventricle. RA: right atrium. RV: right ventricle. 
causes in dogs. In cats, genetic abnormalities in cardiac fibers codifying are important cause of cardiomyopathy [1]. In hamster, dilated and hypertrophic cardiomyopathies are related to the same genetic abnormalities [25].

Considering a previous study that describes a Conepatus chinga as a Trypanosoma cruzi host [22], this infection should be hypothetically considered capable of result in DCM. However, cardiomyopathies or clinical changes were not reported on those animals hosting the parasite [22]. Skunks experimentally infected with T. cruzi showed evidence of chronic granulomatous myocarditis, but minimal clinical signs [24]. In case of Trypanosoma infection, changes in hematological counting would also have been expected instead the normality observed in the present case report.

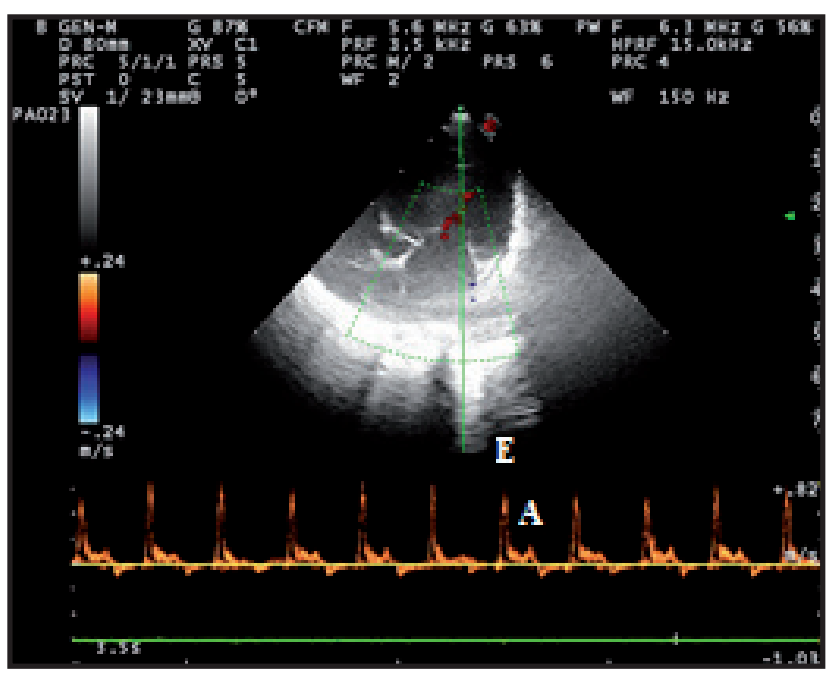

Figure 3. High early (E) e low after (A) diastole waves, left paresternal window, evidencing the restrictive pattern of left ventricle filling, on apical 5 chamber view.
Considering the recent nutritional deficiency suffered by the skunk of this case, the deficiency of taurine was considered as an etiology of DCM. Taurine deficiency can be an important cause of DCM in cats, followed by idiopathic origin [19]. However, taurine serum concentration was earlier referred as normal in a 7-year-old skunk diagnosed with DCM [3], remaining unclear if DCM in skunks could also be caused by taurine deficiency. In the present case, the serum concentration of taurine was not possible to be measured, making hard any conclusion.

Other nutritional impairments were reported in rats as vitamin $E$ or choline deficiency causing cardiac failure or myocardial injury [11]. Moreover, obesity was related to cardiomyopathy in some strains of rats [15] and captivity skunks [17]. In striped skunks, L-carnitine deficiency may also be associated with DCM [3]. As seen above, nutritional aspects may be involved in cardiomyopathies in many rodent species, also dogs and cats [7], prairie dogs [9], woodchucks [2] and African hedgehog [23].

The nutritional role in DCM should still be investigated in skunks. However, it is found that in Andean hog-nosed skunks, up to $85 \%$ of dietary is composed by invertebrates and up to $4 \%$ of small vertebrates [5], making the diet highly proteic, and noticeable different from the nutritional components of the skunk of this case. Although DCN etiologies are not described in the literature, the nutritional, and idiopathic origins were considered in this case, based on clinical history, and complementary evaluations.

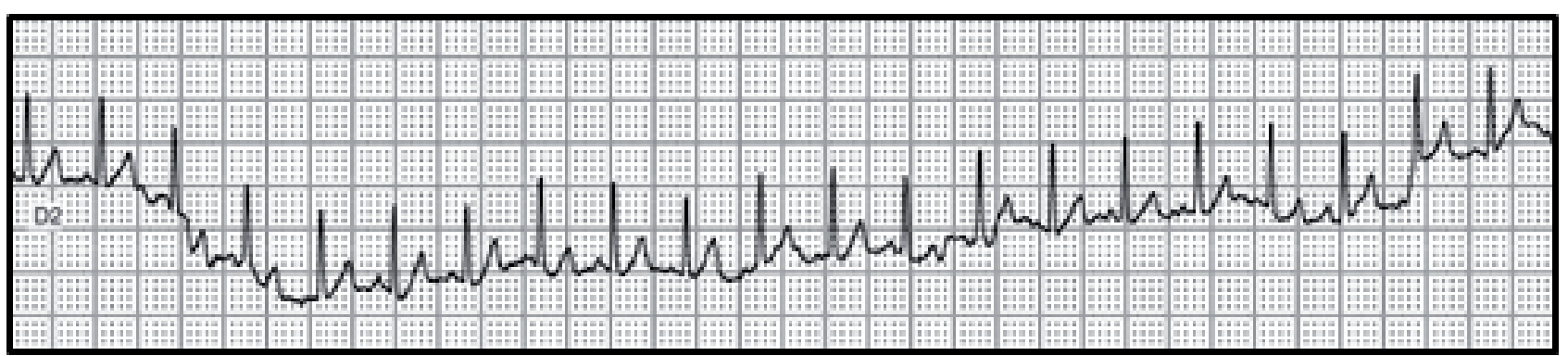

Figure 4. Skunk electrocardiogram registered in a DII derivation, sensitivity N, $25 \mathrm{~mm} / \mathrm{s}$ velocity, showing sinus tachycardia.

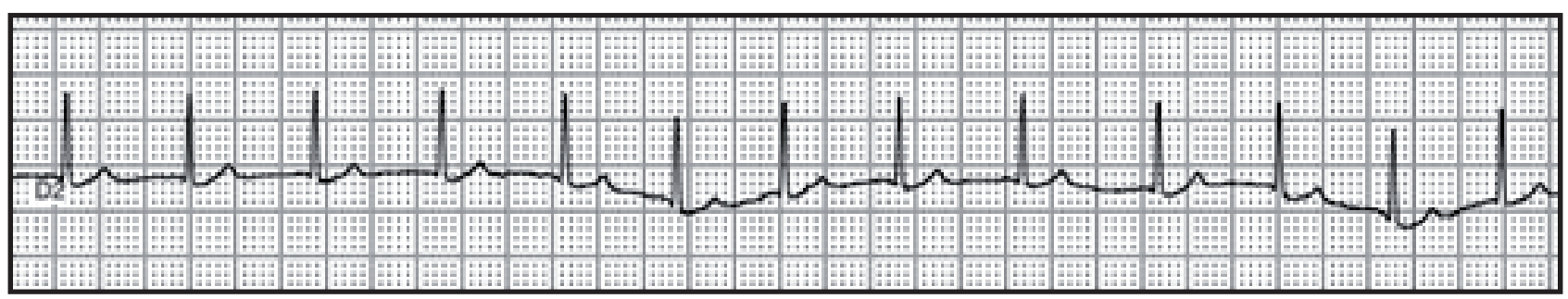

Figure 5. Skunk electrocardiogram registered in a DII derivation, sensitivity $\mathrm{N}, 25 \mathrm{~mm} / \mathrm{s}$ velocity, showing respiratory sinus arrhythmia. 
Table 1. Echocardiographic structural and functional left ventricle variables observed in a skunk (Conepatus chinga) under anesthesia with sevoflurane, showing dilated cardiomyophaty and heart failure, in a day zero and after 5 months of therapy.

\begin{tabular}{|c|c|c|}
\hline Structural variables $(\mathrm{mm})$ & Measures (day zero) & Measures (5 months) \\
\hline Interventricular septum thickness in diastole & 4.0 & 4.8 \\
\hline Left ventricular internal diameter in diastole & 21.5 & 24.1 \\
\hline Left ventricular post wall thickness in diastole & 4.0 & 7.2 \\
\hline Interventricular septum thickness in systole & 4.0 & 4.2 \\
\hline Left ventricular internal diameter in systole & 19.5 & 1.93 \\
\hline Left ventricular post wall thickness in systole & 5.0 & 7.2 \\
\hline Aorta diameter & 4.4 & 7.0 \\
\hline Left atrium diameter & 14.4 & 12.9 \\
\hline $\mathrm{LA} / \mathrm{AO}$ ratio & 3.72 & 1.83 \\
\hline \multicolumn{3}{|l|}{ Functional (systolic and diastolic) variables } \\
\hline Ejection Fraction (\%) & 22 & 43 \\
\hline Fraction shortening $(\%)$ & 9 & 20 \\
\hline Stroke volume (mL) & 3.4 & 8.81 \\
\hline Stroke index $\left(\mathrm{mL} / \mathrm{m}^{2}\right)$ & 33.5 & 45.1 \\
\hline Mitral peak velocity E wave (m/s) & 0.80 & 0.67 \\
\hline Mitral peak velocity A wave (m/s) & 0.16 & 0.33 \\
\hline Mitral E/A ratio & 4.93 & 2.05 \\
\hline Mitral E wave acceleration time (ms) & 28 & --- \\
\hline Mitral E wave deceleration time (ms) & 57.0 & 95.6 \\
\hline Mitral regurgitation velocity $(\mathrm{m} / \mathrm{s})$ & -3.69 & -2.88 \\
\hline Mitral regurgitation gradient $(\mathrm{mmHg})$ & 54.5 & 33.1 \\
\hline Mitral peak velocity E' wave (m/s) & -0.05 & -0.12 \\
\hline Mitral peak velocity A' wave (m/s) & -0.02 & -0.11 \\
\hline Mitral E'/A' ratio & 2.64 & 1.09 \\
\hline Mitral E/E' ratio & -16.33 & -5.58 \\
\hline Tricuspid peak velocity E wave $(\mathrm{m} / \mathrm{s})$ & 0.33 & 0.40 \\
\hline Tricuspid peak velocity A wave $(\mathrm{m} / \mathrm{s})$ & 0.13 & 0.27 \\
\hline Tricuspid E/A ratio & 2.43 & 1.49 \\
\hline Tricuspid regurgitation velocity $(\mathrm{m} / \mathrm{s})$ & -1.6 & none \\
\hline Tricuspid regurgitation gradient $(\mathrm{mmHg})$ & 10.3 & none \\
\hline Aorta peak velocity (m/s) & -0.47 & -1.05 \\
\hline Aorta acceleration time (ms) & 17 & --- \\
\hline Aorta ejection time (ms) & 72.0 & 160.8 \\
\hline Pulmonary peak velocity $(\mathrm{m} / \mathrm{s})$ & -0.31 & -0.84 \\
\hline Pulmonary acceleration time (ms) & 39 & --- \\
\hline Pulmonary artery systolic pressure $(\mathrm{mmHg})$ & 25.3 & --- \\
\hline Isovolumic relaxation time (ms) & 44.0 & 70.3 \\
\hline Left ventricular flow propagation velocity $(\mathrm{m} / \mathrm{s})$ & 0.32 & --- \\
\hline Myocardial performance index & 1.18 & 1.50 \\
\hline
\end{tabular}


Therefore, therapy was initiated, including furosemide at hospital [4 $\mathrm{mg} / \mathrm{kg}$ subcutaneous, single doses] and home [2 $\mathrm{mg} / \mathrm{kg}$ orally, twice a day], enalapril maleate $[0.5 \mathrm{mg} / \mathrm{kg}$ orally, every $48 \mathrm{~h}$ ], taurine [100 $\mathrm{mg}$ per day], and pimobendan [0.5 mg/kg, twice a day]. The dosages were adapted from ferrets [12,28], and well tolerated by the skunk. The patient demonstrated clinical improvement in the second day of therapy, restoring the respiratory status and the level of activity.

Five months after the diagnosis and onset of the therapy, the skunk showed normal growth and level of activity, without any clinical signs of heart failure. Low grade systolic murmur was still noticed. The echocardiographic measurements showed decrease in heart dimensions and improvement on systolic and diastolic function (Table 1). Those findings are also observed in dogs with DCM [8] and myxomatous mitral valve disease [29] treated with pimobendan. The reduction of overload volume and improvement of cardiac global function, imposed by therapy, resulted in absence of tricuspid regurgitation, probably secondary to the decrease of pulmonary arterial and venous pressures.

The electrocardiogram showed respiratory sinus arrhythmia (Figure 5), indicating the restoration of the autonomic balance by the increase of the parasympathetic and decrease of sympathetic tonus after the reduction of the heart failure compensatory mechanisms activation [10].

Considering the good clinical outcome and echocardiographic response to the therapy, a decrease of furosemide was initiated, with frequency of administration to once a day. Pimobendam, taurine, and elanapril prescription were maintained. After 15 days, furosemide was prescribed only every $48 \mathrm{~h}$, follow by complete stop after 20 days. No respiratory distress or activity level decrease was seen until the actual time (30 months after diagnostic).

Most of the cardiovascular diseases reported in skunks are limited to experimental models, wildlife necropsy, and anecdotal reports. Although there are few reports about DCM in skunks, cardiac diseases seemed to be clinically relevant in captivity striped skunk population, especially those from Mephitis gender. No references were found regarding Conepatus gender, so authors believe this is the first report in this species. Future studies will help to properly elucidate the etiology of this disease in skunks. Acquisition of reference values for the species will help to improve the diagnosis. Although challenging due to lack of references for skunks, the diagnostic and therapeutic approach achieved excellent results and should be disclosed.

\section{MANUFACTURERS}

${ }^{1}$ Philips Healthcare. Suzhou, Jiangsu, China.

${ }^{2}$ Carestream Medical and Dental Imaging Systems. Rochester, NY, USA.

${ }^{3}$ Cristália Produtos Químicos e Farmacêuticos Ltda. Itapira, SP, Brazil.

${ }^{4}$ Elsag-Selenia-Ansaldo Officine Toscane Elettromeccaniche Biomedica. Genova, Italy.

${ }^{5}$ Tecnologia Eletrônica Brasileira. São Paulo, SP, Brazil.

${ }^{6}$ Sanofi-Aventis Farmacêutica Ltda. Campinas, SP, Brazil.

${ }^{7}$ Eurofarma Laboratórios S.A. São Paulo, SP, Brazil.

${ }^{8}$ Boehringer Ingelheim do Brasil Química e Farmacêutica Ltda. São Paulo, SP, Brazil.

Declaration of interest. The authors report no conflicts of interest. The authors alone are responsible for the content and writing of paper.

\section{REFERENCES}

1 Abbott J.A. 2010. Feline hypertrophic cardiomyopathy: an update. Veterinary Clinics North America Small Animal Practice. 40: 685-700.

2 Bellezza C.A., Concannon P.W., Hornbucke W.E., Roth L.\& Tennant B.C. 2002. Woochucks as laboratory animals. In: Fox J., Anderson L.C. \& Loew F.M. (Eds). Laboratory Animal Medicine. Boston: Academic Press, pp.309-327.

3 Benato L., Wack A., Cerveny S.N.S., Rosenthal S.L. \& Bronson E. 2014. Survey of cardiac pathologies in captive striped skunks (Mephitis mephitis). Journal Zoo Wildlife Medicine 45: 321-327.

4 Brioni J.D., Varughese S., Ahmed R. \& Bein B. 2017. A clinical review of inhalation anesthesia with sevoflurane: from early research to emerging topics. Journal of Anesthesia. 31(5): 764-778.

5 Donadio E., Martino S.D., Aubone M. \& Novaro A.J. 2004. Feeding ecology of the Andean hog-nosed skunk (Conepatus chinga) in areas under different land use in north-western Patagonia. Journal of Arid Environments. 56: 709-718.

6 Dudás-Györki Z., Szabó Z., Manczur F. \& Vörös K. 2011. Echocardiographic and electrocardiographic examination of clinically healthy, conscious ferret. Journal Small Animal Practice. 52: 18-25.

7 Freeman L.M., Stern J.A., Fries R., Adin D.B. \& Rush J.E. 2018. Diet-associated dilated cardiomyopathy in dogs: what do we know? Journal of American Veterinary Association. 253: 1390-1394. 
8 Fuentes V.L., Corcoran B., French A., Schober K.E., Kleemann R. \& Justus C. 2002. A double-blind, randomized, placebo-controlled study of pimobendan in dogs with dilated cardiomyopathy. Journal of Veterinary Internal Medicine. 16 : 255-261.

9 Funk R. 2004. Medical management of prairie dogs. In: Quesenberry K.E. \& Carpenter J.W. (Eds). Ferrets, Rabbits \& Rodents: Clinical Medicine and Surgery. St. Louis: Saunders, pp.266-273.

10 Giraldo B.F., Pericás M.F., Schröeder R. \& Voss A. 2018. Respiratory sinus arrhythmia quantified with linear and non-linear techniques to classify dilated and ischemic cardiomyopathy. In: Conference Proceedings of the 40th Annual International IEEE Engineering in Medicine and Biology Society. pp.4860-4863.

11 Heatley J.J. 2009. Cardiovascular anatomy, physiology, and disease of rodents and small exotic mammals. Veterinary Clinics of Exotic Animals. 12: 99-113.

12 Hermans K., Geerts T., Cauwerts K., Salheen M.T.S. \& Baert K. 2008. Tolerability of pimobendan in the ferret (Mustela putorius furo). Vlaams Diergeneeskundig Tijdschrift. 78: 53-55.

13 Imamura S. \& Ikeda K. 1987. Comparison of epinephrine-induced arrhythmogenic effect of sevoflurane with isoflurane and halothane. Journal of Anesthesia. 1: 62-68.

14 Kazama T. \& Ikeda K. 1988. The comparative cardiovascular effects of sevoflurane with halotane and isoflurane. Journal of Anesthesia. 2: 63-68.

15 Kohn D.F. \& Clifford C.B. 2002. Biology and diseases of rats. In: Fox J., Anderson L.C. \& Loew F.M. (Eds). Laboratory Animal Medicine. Boston: Academic Press, pp.121-165.

16 Kramer M.H. \& Lennox A. 2003. Exotic pet care: what veterinarians need to know about skunks. Exotic DVM. 5: 36-41.

17 Ledje C. \& Arnason U. 1996. Phylogenetic relationships within caniform carnivores based on analyses of the mitochondrial 12S rRNA gene. Journal of Molecular Evolution. 43: 641-649.

18 Mustonen A.M., Bowman J., Sadowski C., Nituch L.A., Bruce L., Halonen T., Puukka K., Rouvinen-Watt K., Aho J. \& Nieminen P. 2013. Physiological adaptations to prolonged fasting in the overwintering striped skunk (Mephitis mephitis). Comparative Biochemistry and Physiology. 166: 555-563.

19 Novotny M.J., Hogan P.M. \& Flannigan G. 1994. Echocardiographic evidence for myocardial failure induced by taurine deficiency in domestic cats. Canadian Journal of Veterinary Research. 58: 6-12.

20 O'Grady M.R. \& O'Sullivan M.L. 2004. Dilated cardiomyopathy: an update. Veterinary Clinics Small Animal. 34 : 1187-1207.

21 Pascon J.P.E., Pereira Neto G.B., Sousa M.G., Paulino Júnior D. \& Camacho A.A. 2010. Clinical characterization of chronic chagasic cardiomyopathy in dogs. Pesquisa Veterinária Brasileira. 30: 115-120.

22 Pietrokovsky S.M., Schweigmann N.J., Riarte A., Alberti A., Conti O., Montoya S. \& Wisnivesky-Colly C. 1991. The skunk Conepatus chinga as new host of Trypanosoma cruzi in Argentina. Journal of Parasitology. 77: 643-645.

23 Raymond J. \& Garner M. 2000. Cardiomyopathy in captive African hedgehogs. Journal of Veterinary Diagnostic Investigation. 2: 468-472.

24 Ryan C.P., Hughes P.E. \& Howard E.B. 1985. American trypanosomiasis (Chagas' disease) in a striped skunk. Journal of Wildlife Diseases. 21: 175-176.

25 Sakamoto A., Ono K., Abe M., Jasmin G., Eki T., Murakami Y., Masaki T., Toyo-Oka T. \& Hanaoka F. 1997. Both hypertrophic and dilated cardiomyopathies are caused by mutation of the same gene, delta-sarcoglycan, in hamster: an animal model of disrupted dystrophin-associated glycoprotein complex. Proceedings of the National Academy of Sciences of the United States of America. 94: 13873-13878.

26 Schober K.E. \& Chetboul V. 2015. Echocardiographic evaluation of the left diastolic function in cats: hemodynamic determinants and pattern recognition. Journal of Veterinary Cardiology. 17: S102-133.

27 Thomas W.P., Gaber C.E., Jacobs G.J., Kaplan P.M., Lombard C.W., Moise N.S. \& Moses B.L. 1993. Recommendations for standards in transthoracic two-dimensional echocardiography in the dog and cat. Journal of Veterinary Internal Medicine. 7: 247-252.

28 Wagner RA. 2009. Ferret cardiology. Veterinary Clinics of Exotic Animal. 12: 115-134.

29 Wolley R., Smith P., Munro E., Smith S., Swift S., Devine C., Corcoran B. \& French A. 2007. Effects of treatment type on vertebral heart size in dogs with myxomatous mitral valve disease. Internal Journal Applied Research Veterinary Medicine. 5(1): 43-48.

http://seer.ufrgs.br/ActaScientiaeVeterinariae

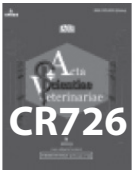

